\title{
Design of Size-Tunable Molecularly Imprinted Polymer for Selective Adsorption of Pharmaceuticals and Biomolecules
}

\section{Shih-Hui Lee ${ }^{1,2}$ and Ruey-An Doong ${ }^{2,3 *}$}

${ }^{1}$ Department of Chemistry, University of California, Irvine, CA, USA

${ }^{2}$ Department of Biomedical Engineering and Environmental Sciences, National Tsing Hua University, 101, Sec. 2, Kuang Fu Road, Hsinchu, 30013, Taiwan

3 Institute of Environmental Engineering, National Chiao Tung University, 1001, University Road, 30010, Hsinchu, Taiwan

\begin{abstract}
The tunable and nano-sized materials have recently been received much attention due to the unique physical and chemical properties which are different from bulk materials. Moleculary imprinted polymer (MIP) nanoparticles with special functions provide opportunities for transformative approaches for a wide of variety applications such as imaging, adsorbent, therapeutic reagents, drug delivery vehicles, sensors, toxin neutralization and enzyme inhibition. In this review, the principle, fabrication approaches, parameters to affect affinity and selectivity of MIP were summarized. The application of MIPs for removal of pollutants as well as the separation of macrobiomolecules was also introduced. Through the tuning processes, MIPs can satisfy the needs of simple, fast, cost-effective and robust. In addition, MIPs are easily to be integrated with the diagnosis and separation system, which have instinct advantages in separation, drug delivery, therapeutic and diagnosis fields. Contents shown in this review clearly show that MIPs are highly potential materials for facile design for not only separating the molecules from small molecules to biomolecules by precipitation polymerization but can also be used for imaging, cell tissue engineering and antidotes.
\end{abstract}

Keywords: Molecularly imprinted polymers (MIPs); Polymerization; Imprinting; Separation; Drug delivery

\section{Introduction}

Polymer materials have been explored for decades and applied in a wide variety of fields such as environment, energy and biomedical purposes. They have many properties such as easy to synthesis, low cost, facile incorporation functional group into resultant polymer and robustness. Recently, the synthesis and exploitation of polymer nanomaterials with novel functionality have been paid much attention in many fields including physics, chemistry, biology, biomedical, pharmaceutical and engineering. Nanomaterials often have unique physical and chemical properties which are different from those of bulk materials. In addition, nanomaterials possess extremely high surface-to-volume ratios which can afford a high capacity for a wide of applications such as adsorption, drug delivery, energy storage and support.

Among the polymeric materials developed, molecularly imprinted polymers (MIPs) are one of the most attractive materials for bioanalytical and biomedical applications. Molecular imprinting technology (MIT) is a promising method to fabricate polymer receptors with nano-size. Although the technique is not very new [1], MIT has been paid much attention in recent years because of the importance of MIPs in novel biomedical applications. Figure 1 shows the fundamental concept of molecularly imprinting process [2]. The print molecule (template) is mixed with the functional monomer selected to interact with specific functionality of the template in step (i). Generally, the template is used to guide the assembly of functional monomers. The template-monomer complex may be formed by noncovalent or covalent associations in step (ii). The complex polymerizes with an excess of cross-linking monomer after adding the initiator in step (iii). A polymerization reaction is then employed to fix the pre-assembled binding groups around the template. Ethylene glycol dimethacrylate (EGDMA) or divinylbenzene (DVB) are commonly used cross-linking monomer. In step (iv), the template is removed from the resultant polymer by solvent extraction or chemical treatment.
The polymer retains a memory for the original template. Imprinted materials can be considered as analogues to enzyme in that binding sites are constrained by three-dimensional scaffold, contains oriented functional groups and can only accommodate guest molecules that fit closely within the cavities [3]. Since the MIT can be tunable to fabricate a wide variety of MIPs for different purposes, this review would focus on the introduction to the principle of MIT, approaches to fabricate MIP nanoparticles, parameters to affect affinity and selectivity, and the selection of template. Finally, the application of MIPs for removal of pollutants as well as the separation of macro biomolecules would also be discussed.
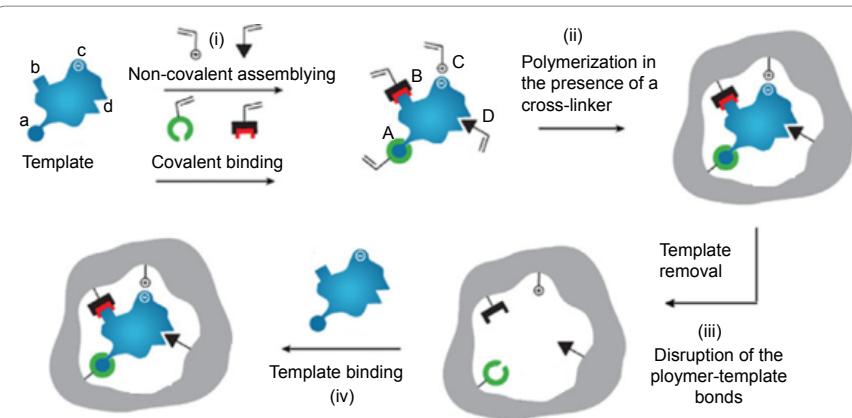

Figure 1: Illustration of molecular imprinting technology processes [2]

*Corresponding author: Ruey-an Doong, Institute of Environmental Engineering National Chiao Tung University, 1001 University Road, Hsinchu, 30010, Taiwan, Tel: +886-3-5712121; Fax: +886-35725958; E-mail: radoong@mx.nthu.edu.tw

Received November 30, 2016; Accepted December 07, 2016; Published December 17, 2016

Citation: Lee SH, Doong RA (2016) Design of Size-Tunable Molecularly Imprinted Polymer for Selective Adsorption of Pharmaceuticals and Biomolecules. J Biosens Bioelectron 7: 228. doi: 10.4172/2155-6210.1000228

Copyright: (c) 2016 Lee SH, et al. This is an open-access article distributed under the terms of the Creative Commons Attribution License, which permits unrestricted use, distribution, and reproduction in any medium, provided the original author and source are credited. 


\section{Strategies of imprinting}

Functional monomers interact with the template in the solutions. The interaction between template and the imprinting polymer includes covalent bonding, non-covalent bonding, and semi-covalent imprinting. Table 1 represents the advantages and disadvantages of imprinting method.

Covalent imprinting: Wulff and his colleagues reported the first covalent imprinting in 1977 [4]. One of the keys factors for successful producing covalent imprinting is the choice of the covalent linkage which connects the functional monomer with a template. These linkages must be stable and reversible. They must be sufficiently stable and be kept intact during the polymerization, but should be easily cleaved later under mild conditions without damaging the imprinting effects [5]. The commonly used linkages include boronic acid esters, acetals, ketals, Schiff bases, disulfide bonds and coordination bonds [6]

Non-covalent imprinting: The non-covalent imprinting method was first reported by Masbach and his colleagues which showed that covalent linkages between functional monomers and templates were not necessarily required for molecular imprinting $[7,8]$. The interactions include hydrogen-bonding, electrostatic, apolar, hydrophobic and other non-covalent bonding $[9,10]$. The major interaction in noncovalent imprinting is hydrogen-bonding.

Semi-covalent imprinting: Whitcombe et al., (1995) have first reported the semi-covalent imprinting in 1995 [11]. The method combines the advantage of covalent bond with anyone of non-covalent bonds. This method involves the covalent attachment during the polymerization and hydrogen-bond formation during the recognition, which could overcome the disadvantages of covalent imprinting.

\section{The polymerization method}

Traditionally, MIPs are prepared by bulk polymerization. This method is, by far, the most widely used for imprinting because of its simplicity and universality. The bulk polymerization is processed by mixing all the components including functional monomer, cross-linker, template and initiator together and then initiates the polymerization reaction by irradiation or by heat. The resultant polymer monoliths are required to be crushed, ground, and sieved to a suitable particle size for the particular purposes such as chromatography and support. However, after these treatments, the morphology of particles is irregular and the binding sites may be crushed to lose the recognition ability. Besides that, there are many disadvantages for bulk polymerization. First of all, bulk polymerization cannot be prepared safely on more than $100 \mathrm{~g}$ due to the exothermic reaction during the polymerization. In addition, polymer particles fabricated in bulk polymerization are wasted up to $70 \%$ during the processes of grinding and sieving [12]. Furthermore, timing consuming and labor intense are drawbacks. Besides these disadvantages, bulk polymerization has limitation to prepare nanosized MIPs. Therefore, several polymerization methods including precipitation, core-shell approaches and emulsion polymerization have been developed to fabricate MIP nanoparticles [13-31].

\begin{tabular}{|l|c|c|c|}
\hline Items & Covalent & Non-covalent & Semi-covalent \\
\hline $\begin{array}{l}\text { Synthesis of monomer-template } \\
\text { conjugate }\end{array}$ & necessary & unnecessary & necessary \\
\hline Polymerization conditions & rather free & Restricted & rather free \\
\hline Removal of template after polymerization & difficult & Easy & difficult \\
\hline Guest - binding and guest-release & slow & Fast & Fast \\
\hline Structure of guest-binding site & clearer & Less clear & Less clear \\
\hline
\end{tabular}

Table 1: Advantages and disadvantages of imprinting method [5].
Precipitation polymerization: This synthesis procedure of precipitation polymerization is based on the method developed by $\mathrm{Ye}$ et al., [13]. The particle size synthesized by precipitation polymerization from very dilute monomer solutions is submicron scale $(0.3-10 \mu \mathrm{m})$. Phase separation occurs during the cross-linking process when the polymeric chains grow more and more insoluble in an organic or aqueous medium. The point of phase separation depends on the nature of MIPs, volume of porogen used and amount of crosslinker employed. In more dilute reaction system, it forms dispersion of macrogel particles in the solvent [14]. It has the advantages that the particle size, shape and morphology can be easily controlled and the polymerization can be performed efficiently in good yield. In addition, precipitate polymerization generates uniform microspheres without any stabilizer or complicated operations [15].

Core-shell approaches: Two common methods, emulsion polymerization and grafting approaches, can be used to fabricate coreshell MIP nanoparticles [2]. The emulsion polymerization is two-step method, the formation of monodisperse seed latex and the creation of molecularly imprinted shell by emulsion polymerization. At first, seed latex can be prepared from a large variety of materials, such as styrene, divinylbenzene, alkyl acrylate, and methacrylate ester-based seeds have all been reported [16-18]. In recent years, magnetic cores are exploited as the core material for recoverable purpose [19-21]. The seed latex particles are monodispersed and vary in size from approximately $30 \mathrm{~nm}$ to more than $1 \mu \mathrm{m}$ in diameter. In the second step, the seed is mixed with another the monomer before the polymerization. The size, morphology, physico-chemical properties of the core-shell polymer particles can be controlled by the composition, structure of monomers and the reaction conditions.

Another method to fabricate MIP core-shell nanoparticles is grafting of the MIP membrane on the seed nanoparticles by controlling living radical polymerization. Atom-transfer radical polymerization (ATRP) [22] and reversible addition-fragmentation chain-transfer polymerization (RAFT) [23] are two common approaches by controlled living radical polymerization. ATRP is a method of forming carboncarbon bond through transition metal catalyst (e.g., $\mathrm{Cu}$ ) [22]. RAFT polymerization uses thiocarbonylthio compounds such as dithioesters, thiocarbomates and xanthates to mediate the polymerization through reversible chain-transfer process [23]. Whatever ATRP or RAFT polymerization, the catalyst or thiocarbonylthio compounds required to initiate polymerization might disrupt the interaction between the template and functional monomer and have to be removed from the resultant polymer nanoparticles.

Besides these two methods, the simplest method is to deposit MIPs membrane on core surface by two steps. First, the core materials are modified by functional groups which the structures end by double bonds. In second step, the core functionalized by double bond was directly mixed with the polymerization solution, including the functional monomer, the cross-linker, the template and initiator with following polymerization. MIPs are coated on core surface to form core-shell structure. Table 2 summarizes the approaches to fabricate the MIP core-shell nanoparticles.

Emulsion polymerization: Emulsion polymerization, one kind of radical polymerization, starts with an emulsion incorporating water, monomer, and surfactant. Surfactants are required in emulsion polymerization. In an oil-in-water emulsion (also called inverse emulsion), the most common type, the reaction system is characterized by the emulsified monomer droplets $(10 \mathrm{~nm}$ to $10 \mathrm{~m})$ dispersed in the continuous aqueous phase with the aid of an oil-in-water surfactant at 
Citation: Lee SH, Doong RA (2016) Design of Size-Tunable Molecularly Imprinted Polymer for Selective Adsorption of Pharmaceuticals and Biomolecules. J Biosens Bioelectron 7: 228. doi: 10.4172/2155-6210.1000228

Page 3 of 9

\begin{tabular}{|c|c|c|c|}
\hline Core & Template & Results & Ref. \\
\hline CdSe/ZnS & Uracil & $\begin{array}{l}\text { [1] Fabrication of MIPs based sensors } \\
\text { [2] The emission intensity increases to several folds when the template binds to the recognition sites of the cross- } \\
\text { linked polymeric matrix. }\end{array}$ & 24 \\
\hline $\mathrm{Fe} 3 \mathrm{O} 4 / \mathrm{Au}$ & Estradiol (E2) & $\begin{array}{l}\text { [1] The magnetic molecularly imprinted polymers were successfully used for detecting E2 in milk power with good } \\
\text { sensitivity, selectivity, reproducibility and efficiency. } \\
\text { [2] The linear range of the imprinted polymer for E2 was } 0.025-10 \mu \mathrm{molL}^{-1} \text { with the limit of detection of } 2.76 \mathrm{nmolL}^{-1}\end{array}$ & 25 \\
\hline $\mathrm{Fe}_{3} \mathrm{O}_{4} / \mathrm{SiO}_{2}$ & Folic acid & $\begin{array}{l}\text { [1] The maximum adsorption capacity of molecularly imprinted polymer was } 8 \mathrm{mg} \mathrm{g}^{-1} \\
\text { [2] The recovery were in the range of } 95 \%-104 \%\end{array}$ & 26 \\
\hline $\mathrm{Fe}_{3} \mathrm{O}_{4} / \mathrm{SiO}_{2}$ & Clozapine & $\begin{array}{l}\text { [1] The magnetic molecularly imprinted based on } \mathrm{Fe}_{3} \mathrm{O}_{4} @ \mathrm{SiO}_{2} \text { were regenerated and their adsorption capacity in } \\
\text { the eighth use showed about an } 6.67 \% \text { loss. } \\
\text { [2] The inta and inter-day precision value were less than } 6 \% \text { and } 4 \% \text {, respectively. }\end{array}$ & 27 \\
\hline $\mathrm{Fe}_{3} \mathrm{O}_{4}$ & $\begin{array}{l}\text { Pesticides (Chlorpyrifos, } \\
\text { Diazinon and Phosalone) }\end{array}$ & $\begin{array}{c}\text { [1] The maximum adsorption capacity for phosalone, diazinon, and chlorpyrifos by Langmuir equation was } 196.07, \\
192.30 \text { and } 172.41 \mathrm{mg} \mathrm{g}^{-1} \\
\text { [2] After four adsorption-desorption cycles, the molecularly imprinted polymers maintained its adsorption capacity } \\
\text { without significant loss. }\end{array}$ & 28 \\
\hline $\mathrm{Fe}_{3} \mathrm{O}_{4} / \mathrm{SiO}_{2}$ & Estradiol & $\begin{array}{l}\text { [1] Reversible addition fragmentation chain transfer polymerization to comprise iron oxide core, a fluorescent dye } \\
\text { layer, and a MIP layer in one system } \\
\text { [2] It can be reused for at least up to } 5 \text { times without significant loss of magnetic moment and signal intensity }\end{array}$ & 29 \\
\hline $\begin{array}{l}\text { Divinylbenzene } \\
\text { styrene }\end{array}$ & $\begin{array}{l}\text { Caffeine/(S)-propranolol/ } \\
\text { theophlline }\end{array}$ & $\begin{array}{l}\text { [1] Emulsion polymerization } \\
\text { [2] } \mathrm{K}_{\mathrm{d}} \text { : caffeine MIP } 200 \mu \mathrm{mol} / \mathrm{dm}^{-3},(\mathrm{~S})-\text { propranolol } 30 \mu \mathrm{mol} / \mathrm{dm}^{-3} \text {, } \\
\text { theophlline } 390 \mu \mathrm{mol} / \mathrm{dm}^{-3}\end{array}$ & 30 \\
\hline
\end{tabular}

Table 2: Summary of MIPs core-shell materials in recent years.

the beginning of polymerization. The droplets are like mini reactor for the polymerization reaction. The particle size is controlled to an extent by the amount (and type) of surfactant present. In addition, when larger amount of surfactant stabilizes a larger interfacial area, smaller particle size is produced. Other parameters, including the initiator concentration and the ratio of functional monomer phase to the continuous phase, also can be adjusted to control the particle size [31]. However, the addition of surfactants may disturb the complex between functional monomer and the template during the polymerization, especially for non-covalent imprinting.

\section{The synthetic parameters affected sensitivity}

Solvents (porogens): The solvent serves first function in dissolving all the components in the polymerization. It serves as the second important function in creating the pores in macroporous polymer [32]. For this reason, it is quite common to refer to the solvent as the "porogen". The solvent can control the morphology and the total pore volume. The porosity and surface area are related with the mass transfer of the substrate. Porogens with low solubility phase separate early and tend to form larger pores and materials with lower surface areas. Conversely, porogens with higher solubility phase separate later in the polymerization providing materials with smaller pore size distributions and greater surface area [33]. In addition, the choice of solvent is more critical in molecular imprinting technology, especially in non-covalent method. The creation of binding sites is corresponding to the formation of the monomer/template complex during pre-polymerization and polymerization steps. The polarity of the porogen affects the formation of the monomer/template complex. Less polar solvents such as chloroform or benzene can facilitate polar non-covalent interaction such as hydrogen bonding or bridging of ionic salts. More polar solvents enhance dissociate the non-covalent interactions in pre-polymer step.

Some studies showed that the solvent optimized when the template binds on MIPs is the same as the porogen used in the polymerization $[34,35]$. The mechanism of template recognition by MIPs originates from two factors. One is the shapes of templates and another is the spatial positioning of the functional groups in the polymer [36]. A swelling process can affect the distance between the groups and the orientation in the polymer. In order to recreate and maintain these shapes and
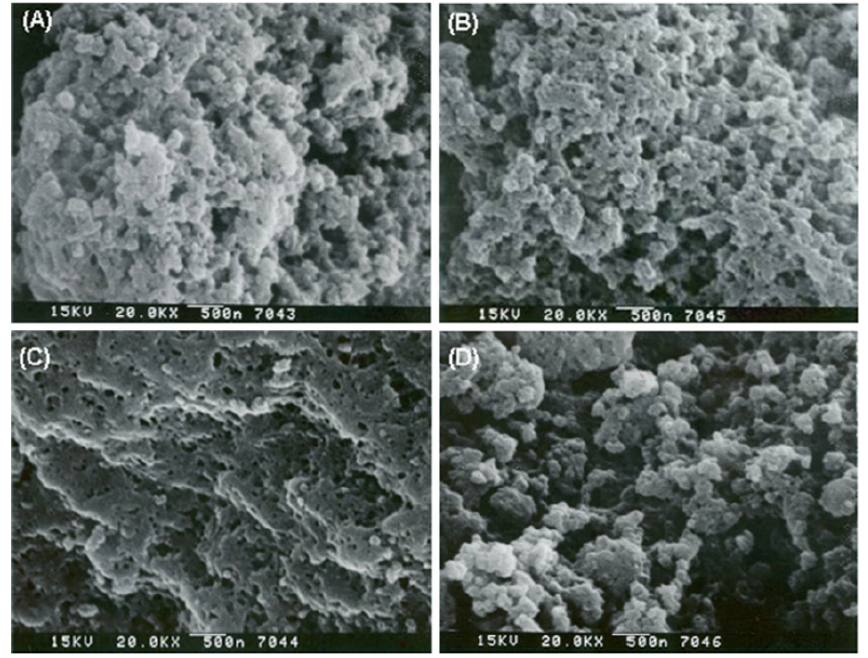

Figure 2: Scanning electron micrographs (SEM) of MIPs prepared in different porogenic solvents. (a) Acetonitrile before the removal of template, (b) acetonitrile after the removal of template, (c) chloroform before the removal of template and (d) chloroform after the removal of template [35].

distance parameters, the optimum rebinding conditions require the same or similar, salvation conditions utilized in polymerization. Lee and Doong have used two porogenic solvents, acetonitrile and chloroform, to prepare MIPs for detection of 17ß-estradiol [35]. After the removal of template by methanol, the MIP structures prepared by both solvent became loose (Figure 2). However, the increase in porosity after the removal of template was also observed, presumably attributed to the removal of template and un-reacted reagents including excess functional monomers, cross-linkers, and linear or branch chain of the functional monomer polymerized with cross-linkers without the connection to network polymers.

Temperature: In principle, MIPs are synthesized by cross-linking complexes of the template and functional monomers. In non-covalent method, the template forms the complex with the functional monomers before the polymerization. The amount ofmonomer/template complexes is relative to the recognition sites and performance. Temperature plays 
an important role in forming monomer/template complexes. The lower temperature polymerization is favorable to the preparation of MIPs based on non-covalent imprinting. High temperature can disrupt the hydrogen bond and electrostatic interaction. Temperature also affects reaction completeness, reaction rates, and polymer structure, such as pore structure and swelling properties. These factors can influent the recognition sites and performance. Lower polymerization temperature is advantageous to stabilize the monomer/template complexes. Higher polymerization temperature is favor to complete the reaction. The affinity and specificity of MIPs are significantly improved by optimizing the polymerization temperature. The temperature also affects the morphology. Pore volume and surface area increase at increasing temperature [37]. The polymer will "memorize" the temperature developed during polymerization [35].

Pressure: Sellergren group shows that the preparation of MIPs at high pressure (1000 bar) can enhance the selectivity [38]. It is because that pressure affects the kinetics of polymerization, the boiling temperature of solvent and the rigidity of polymer. Pressure can affect the kinetics of polymerization reaction which is faster at high pressure as compared with ambient pressure. The boiling temperature of the solvent used as a porogen in the polymerization reaction is affected by the pressure. The polymer made at ambient pressure was more susceptible to the temperature than the polymer at high pressure [39].

The ratio of functional monomer to the template: The formation of the complex between the template and functional monomer with following polymerization is a critical step to create binding sites, especially for non-covalent bond imprinting. The ratio of functional monomer to the template is corresponding to the number of the complex formation. The study shows the ideal ratio to achieve high affinity is 2 to 4 in non-covalent imprinting [33]. Besides, the content of cross-linkers related with hardness of MIPs is concerned due to the maintenance of binding structures.

\section{The selection of the template}

Small molecules imprinted polymers: Molecular imprinting has been applied to small molecule templates in the areas of separations [4042], drug delivery [43,44] and sensors systems [45-47]. In separation purpose, MIPs are usually applied in solid phase extraction $[48,49]$, liquid chromatography [50-52] and capillary electro chromatography $[53,54]$. This part is most close to practical realization. Companies that produce product based on MIPs include MIP Technologies AB (Lund Sweden), POLYIntell (Val de Reuil, France), MIP Solutions Inc. (Kettle Falls, Washington, USA) and Semorex (Fenwood, NJ, USA). Due to high chemical stability and low toxicity, MIPs have advantages to be utilized for drug delivery and antidote [43,44]. In addition to high compatibility with transducers and long-term stability in harsh conditions, MIPs are proper materials to substitute recognizable elements in sensor systems.

Biomolecularly imprinted polymers: Biomolecular targets such as peptides and proteins are the most successful affinity tools used in both fundamental and applied research, such as diagnostics, purification and therapeutics $[55,56]$. Nonetheless, they have inherent disadvantages which include poor chemical, physical, long-term stability, batch-to-batch variability and high cost. MIPs are appropriate materials to substitute biomolecules. Table 3 shows the comparison of biomolecules with MIPs [57]. However, imprinting biomolecules is a challengeable work due to the inherent properties of proteins, such as size, complexity and conformational instability.

Traditional molecularly imprinted polymers (for small molecules) tend to be relatively dense networks in order to retain the binding sites created during the polymerization. Generally, the materials have small pore for diffusion of the template into and out of the matrix. This property leads the problem for biomolecules, such as proteins and antibodies. Biomolecules are entrapped in the network after polymerization and cannot be easily removed to create the binding sites. In addition, they cannot diffuse facilely into the MIPs to find the binding sites. Besides, the polymerization conditions employed for small molecules may lead to the different conformation of biomolecules from those found in the natural environment. Therefore, it may create the recognizable sites which are specific to alternate state. Following re-binding step in natural environmental, MIPs showed non-specific bindings to the template. Some studies shows that three most common biomolecules template (bovine serum albumin, lysozyme, and bovine hemoglobin) in the presence of several common monomers (acrylamide, methacrylic acid, aminophenylboronic acid, acrylic acid, and $\mathrm{N}$-isopropylacrylamide) and crosslinkers (N,N'-methylenebisacrylamide and ethylene glycol dimethacrylate) at relevant polymerization concentrations reveal the conformational change detected by circular dichroism [58,59]. Solvent is also an important issue for imprinting biomolecules. Biomolecules are often insoluble or unstable in aprotic organic solvents generally used for small molecule imprinting. These situations lead biomolecules imprinting to less success than small molecule imprinting. Therefore, only less than $2 \%$ of published work in the area of biomolecules imprinting (Figure 3).

There are four approaches, including bulk imprinting, particlebased imprinting, surface imprinting and epitope imprinting, to fabricate biomolecules imprinted polymers [57]. The removal template to create recognizable sites is a critical problem for biomolecules due to slow mass transfer of large biomolecules from the interior of polymer network, especially bulk imprinting. Bulk imprinting is the imprint of whole macromolecule during the polymerization. As described in previous sections, the processes are time- and labor-intensive due to the requirement of grind and sieve. Therefore, precipitation and emulsion polymerization are utilized to fabricate MIPs nanoparticles (particle-based imprinting) [60-65]. Surface imprinting is the imprint of the template located at or very near surface of polymer. It can be achieved by either synthesizing a thin film using similar approaches as the bulk imprinting or by attaching the template on the surface of substrates with following polymerization. Core-shell approaches are

\begin{tabular}{|c|c|c|}
\hline Parameters & Biomolecules & MIPs \\
\hline Binding affinity & High affinity /specific & Maries \\
\hline Generality & One receptors per analyte & Stable in the harsh conditions (pH, temperature, ionic strength, solvents) \\
\hline Robustness & Limited stability & Inexpensive \\
\hline Cost & Expensive & Long term storage without loss in performance (several months to years) \\
\hline Storage & Days at room temperature & Facile \\
\hline Synthesis/preparation & Time-intensive & Fully compatible \\
\hline
\end{tabular}

Table 3: The comparison of biomolecules with MIPs [55]. 
generally applied for surface imprinting. Surface imprinting facilitates diffusion of biomolecules in and out of MIPs to minimize the template size concerns. Table 4 summarizes current researches in biomolecules imprinting field by surface imprinting [60-65].

Besides surface imprinting, epitope imprinting has the advantage to mitigate the issue about the size of the template. It is the imprint of a short polypeptide during the polymerization and then to recognize the whole polypeptides composed of the certain polypeptides. This technique attempts to more closely mimic the specific interaction between an antibody and antigen. Nishino et al., developed surface imprinting strategy to overcome the mass transfer issue [66]. They immobilized target peptide epitopes on a glass surface then fabricated a polymer thin film on the surface. The thin film harvested by the separation from the glass surface had a high affinity and selectivity for the epitopes and proteins containing the epitopes. The surface imprinting methods solved the mass transfer issue in the target removing process and target rebinding process. However, the materials have limited binding sites due to the surface to volume ratio of the resulting material. Surface imprinting on nanoparticles has intrinsic advantages over bulk counterparts. Nanoparticles (NPs) offer the potential for

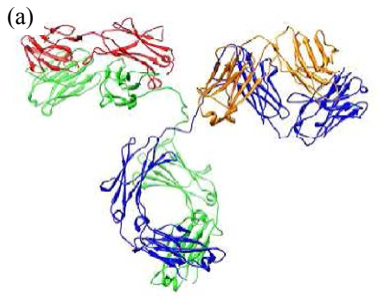

(c)
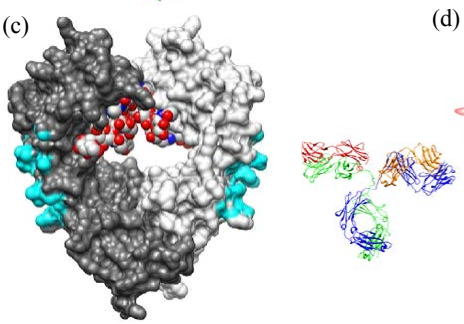

(d)

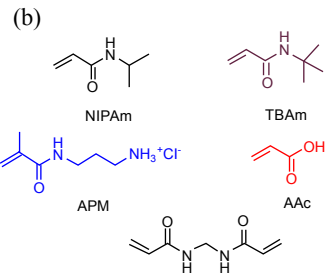

Bis
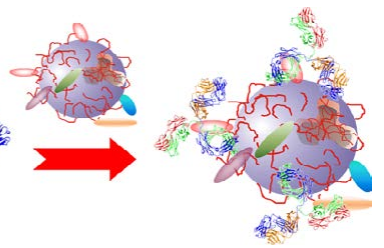

Figure 3: The processes for the preparation of synthetic MIPs for detection of macrobiomolecules of IgG. (a) The structure of IgG with heavy chains in blue and green and light chains in orange and red, (b) monomers used for synthesis of MIP NPs, (c) protein A binding site shown on IgG1-Fc dimer and (d) illustration of detection process of $\operatorname{lgG}[71]$ higher capture capacity and a greater number of end-use applications, such as therapeutic purposes The NP format expands opportunities for applications such as synthetic substitutes for natural antibodies [67-69]. Zeng et al., developed a strategy to create synthetic polymers NPs with antibody-like affinity for a hydrophilic peptide by inverse micro emulsion polymerization [70]. The peptide target was coupled with fatty acid chains of varying chains to orient the imprint peptide at the interface of the water and oil domains during polymerization. The polymer NPs generated by this method had nanomolar affinity and high specificity to target peptides (Table 5).

The engineered synthetic polymer nanoparticles can also be formulated with an intrinsic affinity to a specific domain of a large biomacromolecule. It is noteworthy that the MIPs are usually formed by a kinetically driven process and the sequence of functional monomers in the polymer chain is not controlled. A previous study has used an iterative process to improve affinity by optimizing the composition and proportion of functional monomers [71]. They reported a lightly cross-linked (2\%) $N$-isopropyl acrylamide (NIPAm) synthetic MIPs with diameters of 50-65 nm which can incorporate hydrophobic and carboxylate groups that binds with high affinity to the Fc fragment of IgG. In addition, a computational analysis was used to identify potential NP-protein interaction sites. Candidates include a NP binding domain that overlaps with the protein $\mathrm{A}-\mathrm{Fc}$ binding domain at $\mathrm{pH}$ 5.5. The computational analysis supports the inhibition experimental results and is attributed to the difference in the charged state of histidine residues. Affinity of 3.5-8.5 nm MIPs to the Fc domain at $\mathrm{pH} 5.5$ is comparable to protein $\mathrm{A}$ at $\mathrm{pH} 7$.

\section{Applications}

MIPs are the tailor-made synthetic receptors with the high ability of recognition and binding of target molecules and have recently been demonstrated as the effective materials for a wide variety of applications including separation, solid phase extraction, drug delivery and sensor fabrication. Lee et al., have prepared the MIP particles by precipitation polymerisation to selectively and rapidly capture acetaminophen, a commonly used analgesic and antipyretic drug [75]. By adjusting the ratio of cross-linker and functional monomer, the particle size of MIPs changed from $177 \mathrm{~nm}$ to $2.8 \mathrm{~mm}$ when the ratio increased from 0.43 to 12.8 (Figure 4). In addition, the adsorption equilibrium of acetaminophen by MIPs can be reached within the first $30 \mathrm{~min}$ because of the surface imprinting characteristics and small particle sizes. In addition, the maximum adsorption capacity of acetaminophen and the adsorption constant, well-fitted by Langmuir equation, were 0.35

\begin{tabular}{|c|c|c|}
\hline Templates & Comments & $\operatorname{Re}$ \\
\hline Albumin & $\begin{array}{l}\text { [1] MIP membrane was coated on QCM chip by UV polymerization } \\
\text { [2] Imprinting factor is } 8 \text { and MIPs present selectivity. }\end{array}$ & 60 \\
\hline BSA & $\begin{array}{l}\text { [1] UV polymerization } \\
\begin{array}{l}\text { [2] These polymers exhibited selective recognition of the template relative to competing proteins with up to } 2.9 \text { times more BSA adsorbed than } \\
\text { either glucose oxidase or bovine hemoglobin }\end{array}\end{array}$ & 61 \\
\hline Lysozyme & $\begin{array}{c}\text { [1] A novel monomer, acryloyl- } \beta-C D \text {, to offered hydrophilic exteriorand hydrophobic cavity, was used to self-assemble with the template protein } \\
\text { through hydrogen interaction and hydrophobic interaction. } \\
\text { [2] The column packed with lysozyme imprinted silica beads could effectively separate lysozyme from the mixture. }\end{array}$ & 62 \\
\hline Cyt. C & $\begin{array}{l}\text { [1] Thermal polymerization was used in D.I. water. BSA attached to mica surface for imprinting. } \\
\text { [2] Imprinting factor is up to 4.1. }\end{array}$ & 63 \\
\hline $\mathrm{BHb}$ & $\begin{array}{l}\text { [1] Thermal polymerization in PBS on core-shell superparamagnetic NP. NPs can be recovered by external magnetic field. } \\
\text { [2] The maximum number of imprinted cavities on the surface was } 2.21 \times 10^{18} \mathrm{~g}^{-1} \text {. }\end{array}$ & 64 \\
\hline CEA & $\begin{array}{l}\text { [1] Novel self-assembled monolayers surface MIP for variety of cancer biomarkers. } \\
\text { [2] The CEA assay was both sensitive (detection range } 2.5-250 \mathrm{ng} / \mathrm{mL} \text { ) and specific (no cross-reactivity with hemoglobin; no response by a non- } \\
\text { imprinted sensor). }\end{array}$ & 65 \\
\hline
\end{tabular}

$\mathrm{BHb}$ : Bovine hemoglobin, Cyt C: cytochrome C, CEA: carcinoembryonic antigen, BSA: bovine serum albumin.

Table 4: Summary of biomolecule imprinted polymer by surface imprinting $[55,56]$. 
Citation: Lee SH, Doong RA (2016) Design of Size-Tunable Molecularly Imprinted Polymer for Selective Adsorption of Pharmaceuticals and Biomolecules. J Biosens Bioelectron 7: 228. doi: 10.4172/2155-6210.1000228

Page 6 of 9

\begin{tabular}{|c|c|c|}
\hline Templates & Comments & Ref \\
\hline Angiotensin II, SA (octapeptide) & $\begin{array}{l}\text { [1] IF affected by } \mathrm{pH} \text {, ionic strength of PBS and } \% \text { acetonitrile in aqueous loading solution } \\
\text { [2] MIPs column: detection limit of } 8 \text { pmol and a response that is linear }\left(r^{2}>0.99\right) \text { over the concentration range } \\
0.4-20 \mu \mathrm{M} \text {. }\end{array}$ & 72,73 \\
\hline $\begin{array}{l}15 \text { mer peptide for dengue virus } \\
\text { protein }\end{array}$ & $\begin{array}{l}\text { [1] Fabrication of MIP membrane on QCM chip by UV polymerization } \\
\text { [2] The thickness is } 70 \mathrm{~nm} \text {. The selectivity for 15-mer peptide and parent protein in patient samples represents. }\end{array}$ & 74 \\
\hline Epitopes for Cyt c, ADH and BSA & $\begin{array}{l}\text { [1] C-terminus nonapeptide of Cyt c, ADH and BSA were used as the imprinting peptides. } \\
\text { [2] Specificity to target protein. When it changed one amino acid to imprint, it shows no binding }\end{array}$ & 66 \\
\hline Nanopeptide sequence for GPF & $\begin{array}{l}\text { [1] A method for the fabrication of synthetic polymer NPs with surface binding sites for hydrophilic peptides by } \\
\text { inverse emulsion polymerization } \\
\text { [2] By surveying peptides with fatty acid chains of varying length, conditions were found to achieve success peptide } \\
\text { imprinting at the droplet interface }\end{array}$ & 71 \\
\hline
\end{tabular}

Cyt C: cytochrome C, BSA: bovine serum albmin, ADH: alchol dehydrogenase, GPF: green fluorescent protein.

Table 5: Summary of biomolecule imprinted polymer by epitope imprinting.

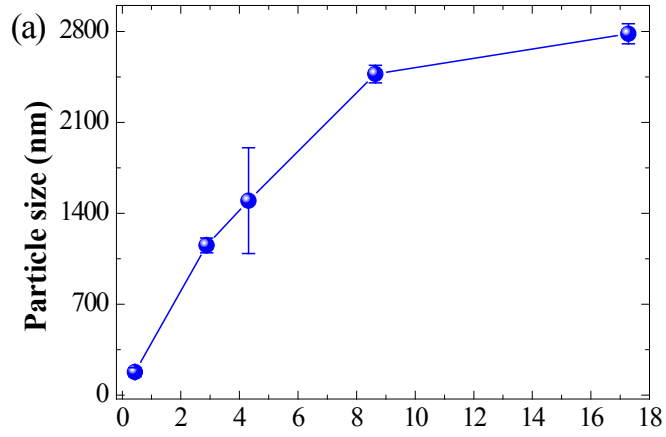

Cross-linker/Functional monomer (molar ratio)

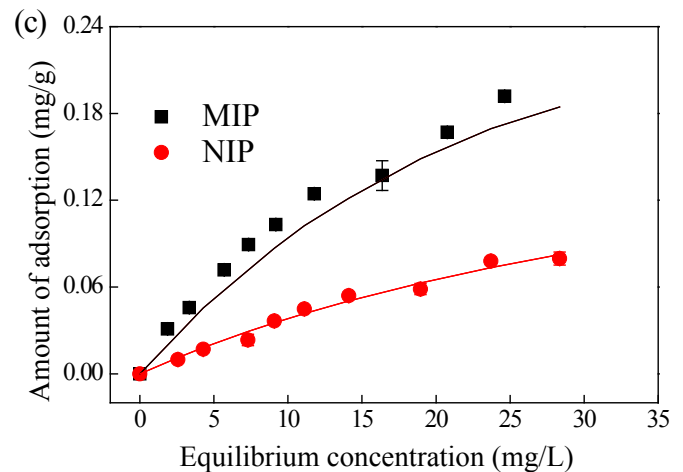

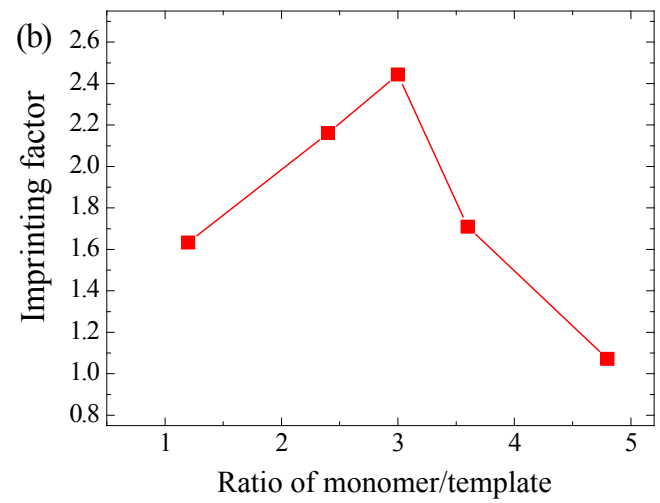

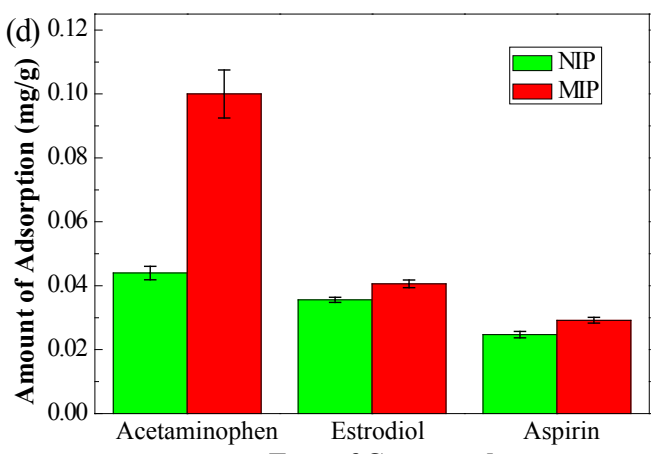

Type of Compounds

Figure 4: The (a) particle size as a function of C/F ratio of the acetaminophen-imprinted polymers (A-MIPs), (b) the imprinting factor of A-MIPs synthesised at various ratios of functional monomer to template ranging from 1.2 to 4.8 , (c) adsorption isotherm of A-MIPs and non-imprinted polymers (NIP) toward acetaminophen adsorption and (d) the selective adsorption of acetaminophen, estradiol and aspirin on A-MIPs and NIPs [75].

$\mathrm{mg} / \mathrm{g}$ and $0.045 \mathrm{~L} / \mathrm{mg}$. In addition, the MIPs exhibited the excellent selectivity to acetaminophen. The MIPs prepared in chloroform also found to have a higher adsorption capacity towards $17 \AA$-estradiol than that in acetonitrile. When using capacity factor values to understand the selectivity of MIPs for similar structures of compounds, the estradioltemplated MIPs show good selective toward the templates and the rebinding ability from selective recognition sites of MIPs decreased in the order $17 ß$-estradiol $>$ testosterone $>$ benzo[a]pyrene $>$ progesterone $>$ phenol, clearly showing that MIPs can be used to selectively adsorb as well as to separate chemicals with similar structures.

Pang et al., reported a novel system for harvesting cell sheets which relied on poly( $N$-isopropyl-acryamide) (PNIPAAm)-based MIP hydrogel layer with thermo-responsive affinity toward specific biomolecules by redox-initiated polymerization [76]. Molecular imprinting methodology was to introduce the cell-adhesive peptide Arg-Gly-Asp-Ser (RGDS) onto a thermo-responsive cell culture substrate, which was innovatively used as a highly efficient novel system for harvesting cell sheets. With the reversible thermoresponsive specific recognition sites, the imprinted hydrogel could recognize and bind RGDS molecules at a common temperature for cell culture $\left(37^{\circ} \mathrm{C}\right)$, and then rapidly release RGDS when the temperature was lowered. The imprinted thermo-responsive hydrogel proved to not only promote cell adhesion during cell culture, but also facilitate cell detachment in cell sheet harvest process. This method solved the longstanding problem in cell sheet technology.

Kunath et al., reported the application of MIP nanoparticles labeled by fluorescent for cell and tissue imaging [77]. Glucuronic acid which is abundant on the surface of cells such as keratinocytes in 
(a)

(b)

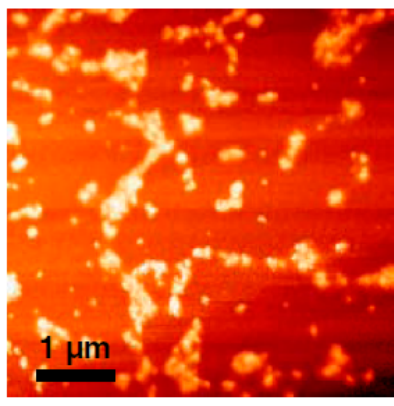

(c)

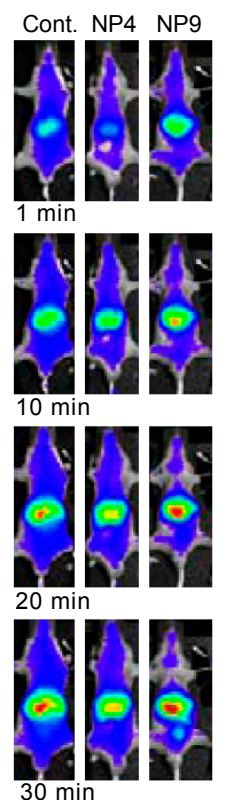

Figure 5: Interaction between melittin and MIP synthesized with various feed ratios of tert-butyl groups (TBAm) and carboxylic acids. (A) AFM image of MIP NPs. (b) Survival rates of mice over a $24 \mathrm{~h}$ period after intravenous injection of $4.5 \mathrm{mg} \mathrm{kg}^{-1}$ melittin (green). $30 \mathrm{mg} \mathrm{kg}^{-1}$ of melittin imprinted NPs (red), not imprinted NPs (gray) was systemically administrated via a tail vain 20 seconds after melittin injection. $\mathrm{P}$ Values are calculated by the Wilcoxon test. (c) Fluorescent images of Cy5-melittin (70 min after injection) in livers from mice with various doses of Cy5-melittin and molecularly imprinted nanoparticles [81]

form of hyaluronan as part of the glyocalix was chosen as the target for epitope imprinting. This strategy demonstrated that MIPs as "plastic antibodies" have a great potential for bioimaging and multiple labels are more easily implemented for staining several targets. Panagiotopulou et al., reported a novel photopolymerization method to coat quantum dots (QDs) with molecularly imprinted polymer shells by using the visible light emitted from QDs excited by UV light [78]. Glucuronic and $\mathrm{N}$-acetylneuraminic acid were imprinted, respectively, on QDs to recognize hyaluronic acid and sialylated glycoproteins and glycolipids on keratinocytes and demonstrate the multiplexed detection and imaging of glycosylations in cells.

Hoshino et al., designed a novel synthetic NPs that bind and suppress the activity of a toxic peptide. Melittin, a 26 amino acid peptide isolated from bee venom, is a representative of membrane-damaging toxins [67]. It was selected as the target to demonstrate the concept by neutralizing toxin. A small library of $\mathrm{N}$-isopropylacrylamide (NIPAm) based copolymer nanoparticles incorporating combinations of functional monomers were prepared by precipitation polymerization. $\mathrm{N}$-tert-Butylacrylamide (TBAm), acrylamide (AAm), $\mathrm{N}$-(3aminopropyl) methacrylamide hydrochloride (3-APM) and acrylic acid (AA) were used as hydrophobic, hydrophilic, positively charged and negatively charged functional monomers, respectively. These functional monomers were used to mimic antibodies, like 23 amino acids in nature. In vitro experiment, Hoshino et al., reported that NPs can capture and clear Melittin in bloodstream of living mice. High binding capacity and selectivity of NPs comparable to nature antibodies were achieved by combining a functional monomer optimization strategy and imprinting process. The mortality and peripheral toxic symptoms of melittin were significantly diminished and the injection of NPs can increase survival rate. The melittin.MIPNP complexes are then cleared from the blood by the mononuclear phagocytic system in the liver (Figure 5) [79-81]. These results provide a platform to design plastic antidotes and reveal the potential and possible limitations of using synthetic polymer nanoparticles as plastic antidotes.

\section{Conclusions}

In this review, we have given a short introduction from the development of MIPs to the applications in selective adsorption of small molecules of pharmaceuticals and separation of macromolecules. MIPs have been developed to satisfy the need for simple, fast, cost-effective and robust purposes. In addition, MIPs are easily to be integrated with the diagnosis and separation system. It is obvious that MIPs (artificial antibodies) have instinct advantages in separation, drug delivery, therapeutic and diagnosis fields. MIPs show high potential not only in these areas but also in imaging, cell tissue engineering and antidotes. We believe that molecular imprinting is a promising technique in the future.

\section{Acknowledgements}

The authors thank the Ministry of Science and Technology (MOST), Taiwan for financial support under grant Nos. MOST 104-2221-E-009-020-MY3 and 105-2113-M-009-023-MY3.

\section{References}

1. Polyakov MV (1931) Adsorption properties and structure of silica gel. Zhur Fiz Khim 2: 799-805

2. Poma A, Turner APF, Piletsky SA (2010) Advances in the manufacture of MIP nanoparticles. Trends Biotechnol. 28: 629-637.

3. Alexander C, Davidson L, Hayes W (2003) Artificial molecular recognition materials with applications in synthesis and catalysis. Tetrahedron 59: 2025-2057.

4. Wulff G, Grobeeinsler R, Vesper W, Sarhan A (1997) Enzyme-analogue built polymers, 5 . On the specificity distribution of chiral cavities prepared in synthetic-polymers. Makromol Chem 178: 2817-2825.

5. Komiyama M, Takeuchi T, Takashi M, Hiroyuki A (2002) Molecular Imprinting From Fundamentals to Applications. Wiley-Vch pp: 159.

6. Wulff G, Bellmann S, Schmid J, Podzimek S (1997) Preparation and polymeranalogous reactions of a poly(vinyl sugar) with a $C-C$ bond between sugar and polymer backbone. Macromol Chem Physic 198: 763-775.

7. Vlatakis G, Andersson L, Muller R, Mosbach K (1993) Drug assy using antibody mimics made by molecular imprinting. Nature 361: 645-647.

8. Arshady R, Mosbach K (1981) Synthesis of substrate-selective polymers by host-guest polymerization. Macromol Chem Phys 182: 687-692.

9. Piletsky S, Andersson H, Nicholls I (1999) Combined hydrophobic and electrostatic interaction-based recognition in molecularly imprinted polymers. Macromolecules 32: 633-636.

10. Cui A, Singh A, Kaplan D (2002) Enzyme-based molecular imprinting with metals. Biomacromolecules 3: 1353-1358.

11. Whitcombe M, Rodriguez M, Villar P, Vulfson E (1995) A new method for the introduction of recognition site functionality into polymers prepared by molecular imprinting - synthesis and characterization of polymeric receptors for cholesterol. J Am Chem Soc 117: 7105-7111.

12. Perez-Moral N, Mayes A (2004) Comparative study of imprinted polymer particles prepared by different polymerisation methods. Anal Chim Acta 504 $15-21$

13. Ye L, Cormack P, Mosbach K (1999) Molecularly imprinted monodisperse microspheres for competitive radioassay. Anal Commun 36: 35-38.

14. Ye L, Cormack P, Mosbach K (2001) Molecular imprinting on microgel spheres. Anal Chim Acta 435: 187-196.

15. Ye L, Mosbach K (2001) Molecularly imprinted microspheres as antibody binding mimics. React Funct Polym 48: 149-157.

16. Carter S, Rimmer S (2004) Surface molecularly imprinted polymer core-shell particles. Adv Funct Mater 14: 553-561. 
Citation: Lee SH, Doong RA (2016) Design of Size-Tunable Molecularly Imprinted Polymer for Selective Adsorption of Pharmaceuticals and Biomolecules. J Biosens Bioelectron 7: 228. doi: 10.4172/2155-6210.1000228

Page 8 of 9

17. Perez N, Whitcombe M, Vulfson $E$ (2001) Surface imprinting of cholesterol on submicrometer core-shell emulsion particles. Macromolecules 34: 830-836.

18. Perez N, Whitcombe M, Vulfson E (2000) Molecularly imprinted nanoparticles prepared by core-shell emulsion polymerization. J Appl Polym Sci 77: 18511859.

19. Jin GY, Li W, Yu SN, Peng YY, Kong JL (2008) Novel superparamagnetic core-shell molecular imprinting microspheres towards high selective sensing Analyst 133: 1367-1372.

20. Zhou WH, Lu CH, Guo XC, Chen FR, Yang HH, et al. (2010) Mussel-inspired molecularly imprinted polymer coating superparamagnetic nanoparticles for protein recognition. J Mater Chem 20: 880-883.

21. Tan CJ, Tong YW (2007) Preparation of super paramagnetic ribonuclease a surface-imprinted submicrometer particles for protein recognition in aqueous media. Anal Chem 79: 299-306.

22. Matyjaszewski K, Xia JH (2001) Atom transfer radical polymerization. Chem Rev 101: 2921-2990.

23. Moad G, Chong YK, Postma A, Rizzardo E, Thang SH (2005) Advances in RAFT polymerization: the synthesis of polymers with defined end-groups. Polymer 46: 8458-8468.

24. Lin Cl, Joseph AK, Chang CK, Lee YD (2004) Synthesis and photoluminescence study of molecularly imprinted polymers appened onto $\mathrm{Cd} / \mathrm{ZnS}$ core-shell. Biosens Bioelectron 20: 127-131.

25. Han Q, Shen X, Zhu W, Zhu C, Zhou X, Jiang H (2016) Magnetic sensing film based on $\mathrm{Fe}_{3} \mathrm{O}_{4} @ A$ Au-GSH molecularly imprinted polymers for the electrochemical detection of estradiol. Biosens Bioelectron 79: 180-186.

26. Hussain S, Khan S, Gul S, Pividori MI, Sotomayor MTD (2016) A novel core@ shell magnetic molecular imprinted nanoparticles for selective determination of folic acid in different food samples. React Funct Polym 106: 51-56.

27. Javidi J, Esmaeilpour M, Khansari MR (2015) Synthesis, characterization and application of core-shell magnetic molecularly imprinted polymers for selective recognition of clozapine from human serum. RSC Adv 5: 73268- 73278

28. Masoumi A, Hemmati K, Ghaemy M (2016) Recognition and selective adsorption of pesticides by superparamagnetic molecularly imprinted polymer nanospheres. RSC Adv 6: 49401-49410.

29. Li Y, Dong CK, Chu J, Qi JY, Li X (2011) Surface molecular imprinting onto fluorescein-coated magnetic nanoparticles via reversible addition fragmentation chain transfer polymerization: a facile three-in-one system for recognition and separation of endocrine disrupting chemicals. Nanoscale 3: 280-287.

30. Carter SR, Rimmer S (2004) Surface molecularly imprinted polymer core-shel particles, Adv Funct Mater 14: 553-561.

31. Harkins WD (1947) A general theory of the mechanism of emulsion polymerization. J. Am Chem Soc 69: 1428-1444.

32. Cormack P, Elorza A (2004) Molecularly imprinted polymers: synthesis and characterization. J Chromatogr B 804: 173-182.

33. Spivak DA (2005) Optimization, evaluation, and characterization of molecularly imprinted polymers. Adv Drug Deliver Rev 57: 1779-1794.

34. Piletsky S, Piletska E, Karim K, Freebairn K, Legge C Turner (2002) Polymer Cookery: Influence of Polymerization Conditions on the Performance of Molecularly Imprinted Polymers. Macromolecules 35: 7499-7504.

35. Lee SH, Doong RA (2012) Adsorption and selective recognition of 17 $\beta$-estradiol by molecularly imprinted polymers. J Polym Res 19: 9939.

36. Spivak D, Simon R, Campbell J (2004) Evidence for shape selectivity in noncovalently imprinted polymers. Anal Chim Acta 504: 23-30.

37. Lu Y, Li C, Wang X, Sun P, Xing X (2004) Influence of polymerization temperature on the molecular recognition of imprinted polymers. J Chromatogr B 804: 53-59.

38. Sellergren B, Dauwe C, Schneider T (1997) Pressure-Induced Binding Sites in Molecularly. Imprinted Network Polymers. Macromolecules 30: 2454-2459.

39. Piletsky S, Guerreiro A, Piletska E, Chianella I, Karim K, Turner A (2004) Polymer Cookery. 2. Influence of Polymerization Pressure and Polymer Swelling on the Performance of Molecularly Imprinted Polymers. Macromolecules 37: 50185022

40. Tamayo FG, Turiel E, Martin-Esteban A (2007) Molecularly imprinted polymers for solid-phase extraction and solid-phase microextraction: Recent developments and future trends. J Chromatogr A 1152: 32-40.

41. Caro E, Marce RM, Borrull F, Cormack PAG, Sherrington DC (2006) Application of molecularly imprinted polymers to solid-phase extraction of compounds from environmental and biological samples. Trac-Trend Anal Chem 25: 143-154.

42. He CY, Long YY, Pan JL, Li K, Liu F (2007) Application of molecularly imprinted polymers to solid-phase extraction of analytes from real samples. J Biochem Bioph Meth 70: 133-150.

43. Sellergren B, Allender CJ (2005) Molecularly imprinted polymers: A bridge to advanced drug delivery. Adv Drug Deliv Rev 57: 1733-1741.

44. Cunliffe D, Kirby A, Alexander C (2005) Molecularly imprinted drug delivery systems. Adv Drug Deliv Rev 57: 1836-1853.

45. Fuchs Y, Soppera O, Haupt K (2012) Photopolymerization and photostructuring of molecularly imprinted polymers for sensor applications-a review. Anal Chim Acta 717: 7-20.

46. Ouyang RZ, Lei JP, Ju HX (2010) Artificial receptor-functionalized nanoshell: facile preparation, fast separation and specific protein recognition. Nanotechnology 21: 185502.

47. Yan S, Fang Y, Gao Z (2007) Quartz crystal microbalance for the determination of daminozide using molecularly imprinted polymers as recognition element Biosens Bioelectron 22: 1087-1091.

48. Qiao FX, Sun HW, Yan HY, Row KH (2006) Molecularly Imprinted Polymers for Solid Phase Extraction. Chromatographia 64: 625-634.

49. Lasakova M, Jandera P (2009) Molecularly imprinted polymers and their application in solid phase extraction. J Sep Sci 32: 799-812.

50. Hoshina K, Horiyama S, Matsunaga H, Haginaka J (2009) Simultaneous Determination of Antiepileptics in River Water Samples by Liquid Chromatography-Tandem Mass Spectrometry. J Chromatogr A 1216: 49574962

51. Chen LG, Zhang XP, Xu Y, Du XB, Sun X, et al. (2010) Determination of fluoroquinolone antibiotics in environmental water samples based on magnetic molecularly imprinted polymer extraction followed by liquid chromatographytandem mass spectrometry. Anal Chim Acta 662: 31-38.

52. Mullett WM, Walles M, Levsen K, Borlak J, Pawliszyn J (2004) Multidimensiona on-line sample preparation of verapamil and its metabolites by a molecularly imprinted polymer coupled to liquid chromatography-mass spectrometry. J Chromatogr B-Analytical Technologies in the Biomedical and Life Sciences 801: 297-306.

53. Ou JJ, Li X, Feng S, Dong J, Dong XL, et al. (2007) Preparation and Evaluation of a Molecularly Imprinted Polymer Derivatized Silica Monolithic Column for Capillary Electrochromatography and Capillary Liquid Chromatography. Anal Chem 79: 639-646.

54. Spegel P, Schweitz L, Nilsson S (2003) Molecularly imprinted polymers in capillary electrochromatography: Recent developments and future trends. Electrophoresis 24: 3892-3899.

55. Wackerlig J, Schirhagl R (2016) Applications of Molecularly Imprinted Polymer Nanoparticles Their Advances toward Industrial Use: A Review. Anal Chem 88 250-261.

56. Chen W, Ma Y, Pan J, Meng Z, Pan G, Sellergren B (2015) Molecularly Imprinted Polymers with Stimuli-Responsive Affinity: Progress and Perspectives. Polymers 7: 1689-1715.

57. Kryscio DR, Peppas NA (2012) Critical review and perspective of macromolecularly imprinted polymers. Acta Biomater 8: 461-473.

58. Kryscio DR, Fleming MQ, Peppas NA (2012) Conformational studies of common protein templates in macromolecularly imprinted polymers. Biomed Microdevices 14: 679-687.

59. Kryscio DR, Fleming MQ, Peppas NA (2012) Protein conformational studies for macromolecularly imprinted polymers. Macromole Biosci 12: 1137-1144.

60. Lin TY, Hu CH, Chou TC (2004) Determination of albumin concentration by MIP-QCM sensor. Biosens Bioelectron 20: 75-81.

61. Kryscio DR, Peppas NA (2012) Surface imprinted thin polymer film systems with selective recognition for bovine serum albumin. Anal Chim Acta 718: 109-115.

62. Zhang W, Qin L, He XW, Li WY, Zhang YK (2009) Novel surface modified 
Citation: Lee SH, Doong RA (2016) Design of Size-Tunable Molecularly Imprinted Polymer for Selective Adsorption of Pharmaceuticals and Biomolecules. J Biosens Bioelectron 7: 228. doi: 10.4172/2155-6210.1000228

molecularly imprinted polymer using acryloyl-beta-cyclodextrin and acrylamide as monomers for selective recognition of lysozyme in aqueous solution. $J$ Chromatogr A 1216: 4560-4567.

63. El Kirat K, Bartkowski M, Haupt K (2009) Probing the recognition specificity of a protein molecularly imprinted polymer using force spectroscopy. Biosens Bioelectron 24: 2618-2624.

64. Ouyang RZ, Lei JP, Ju HX (2010) Artificial receptor-functionalized nanoshell: facile preparation, fast separation and specific protein recognition. Nanotechnology 21: 185502.

65. Wang YT, Zhang ZQ, Jain V, Yi JJ, Mueller S, et al. (2010) Potentiometric sensors based on surface molecular imprinting: Detection of cancer biomarkers and viruses. Sensor Actuat B-Chem 146: 381-387.

66. Nishino H, Huang CS, Shea KJ (2006) Selective protein capture by epitope imprinting. Angew Chem Int Edit 45: 2392-2396.

67. Hoshino Y, Kodama T, Okahata Y, Shea KJ (2008) Peptide Imprinted Polymer Nanoparticles: A Plastic Antibody. J Am Chem Soc 130: 15242-15243.

68. Li Y, Yang HH, You QH, Zhuang ZX, Wang XR (2006) Protein Recognition via Surface Molecularly Imprinted Polymer Nanowires. Anal Chem 78: 317-320.

69. Tan CJ, Wangrangsimakul S, Bai R, Tong YW (2008) Defining the Interactions between Proteins and Surfactants for Nanoparticle Surface Imprinting through Mini emulsion Polymerization. Chem Mater 20: 118-127.

70. Zeng ZY, Hoshino Y, Rodriguez A, Yoo HS, Shea KJ (2010) Synthetic Polymer Nanoparticles with Antibody-like Affinity for a Hydrophilic Peptide. ACS Nano 4: 199-204.

71. Lee SH, Hoshino Y, Randall A, Zeng ZY, Baldi P, et al. (2012) Engineered synthetic polymer nanoparticles as IgG affinity ligands. J Am Chem Soc 134: 15765-15772.

72. Rachkov A, Hu MJ, Bulgarevich E, Matsumoto T, Minoura N (2004) Molecularly imprinted polymers prepared in aqueous solution selective for [Sar(1),Ala(8)] angiotensin II. Anal Chim Acta 504: 191-197.

73. Rachkov A, Minoura N (2000) Recognition of oxytocin and oxytocin-related peptides in aqueous media using a molecularly imprinted polymer synthesized by the epitope approach. J Chromatogr A 889: 111-118.

74. Tai DF, Lin CY, Wu TZ, Chen LK (2005) Recognition of Dengue Virus Protein Using Epitope-Mediated Molecularly Imprinted Film. Anal Chem 77: 5140-5143.

75. Lee SH, Ong HL, Doong RA (2016) Design of size-tunable molecularly imprinted polymer for selective adsorption of acetaminophen. Clean Technol Environ Policy pp: 1-8

76. Pan G, Guo Q, Ma Y, Yang H, Li B (2013) Thermo-Responsive Hydrogel Layers Imprinted with RGDS Peptide: A System for Harvesting Cell Sheets. Angew Chem Int Ed 52: 6907-6911.

77. Kunath S, Panagiotopoulou M, Maximilien J, Marchyk N, Sänger J, et al. (2015) Cell and Tissue Imaging with Molecularly Imprinted. Adv Healthcare Mater 4 $1322-1326$.

78. Panagiotopoulou M, Salinas Y, Beyazit S, Kunath S, Duma L, et al. (2016) Molecularly Imprinted Polymer Coated Quantum Dots for Multiplexed Cell Targeting and Imaging. Angew Chem Int Ed 55: 8244-8248.

79. Hoshino Y, Urakami T, Kodama T, Koide H, Oku N, et al. (2009) Design of Synthetic Polymer Nanoparticles that Capture and Neutralize a Toxic Peptide. Small 5: 1562-1568.

80. Hoshino $\mathrm{Y}$, Koide H, Urakami T, Kanazawa H, Kodama T, et al. (2010) Recognition, neutralization and clearance of target peptides in the blood stream of living mice by molecular imprinted polymer nanoparticles: a plastic antibody. J Am Chem Soc 132: 6644-6645.

81. Hoshino $Y$, Koide H, Furuya K, Haberaecker WW, Lee SH, et al. (2012) The rational design of a synthetic polymer nanoparticle that neutralizes a toxic peptide in vivo. Proc Natl Acad Sci 109: 33-38. 